\title{
Optical Properties of Planar Chiral Meta-Materials
}

\author{
A. Potts, A. Papakostas ${ }^{1}$, N.I. Zheludev, H.J. Coles, R. Greef ${ }^{2}$ and D.M. Bagnall ${ }^{1}$ \\ Department of Physics and Astronomy, University of Southampton, Highfield, \\ Southampton SO17 1BJ, UK \\ ${ }^{1}$ Department of Electronics and Computer Science, University of Southampton, \\ ${ }^{2}$ Department of Chemistry, University of Southampton,
}

\begin{abstract}
The polarization state of visible light is found to be altered upon reflection from artificial two-dimensional chiral media. Arrays of metallic planar chiral structures were fabricated by electron beam lithography and ion beam milling. The characteristic dimensions on the chiral elements correspond to wavelengths in the near-IR. Our chiral media are found to induce strong polarization effects, with the handedness of individual elements having a direct effect on the sense and magnitude of rotation of the diffracted light.
\end{abstract}

\section{INTRODUCTION}

The ability of left- and right-handed asymmetrical (chiral) three-dimensional helical molecules to rotate the polarization state of light (known as optical activity) is one of the cornerstones of modern optics. However, this concept of chirality also exists in two-dimensions [1], a planar object is said to be chiral if it cannot be brought into congruence with its mirror image unless it is lifted from the plane. The question as to whether planar medium could affect the polarization state of light, in a manner similar to three-dimensional chiral molecules, has so far remained unanswered. Recently, there has been considerable theoretical interest in this subject, with many authors [2-4] proposing planar structures with chirality that could exhibit selective electromagnetic properties. Now, using electron beam lithographic techniques, we have manufactured left and right-handed forms of an artificial medium consisting of microscopic chiral metallic objects distributed regularly in a plane. In this artificial medium we have observed optical manifestations of planar chirality in the form of handedness-sensitive rotations of the polarization state and elliptization of visible light diffracted from the chiral structures.

\section{DEVICE DESIGN AND FABRICATION PROCESS}

The artificial two-dimensional media that we have created consist of microscopic chiral metallic objects (gammadions) that are arranged in regular two-dimensional square gratings to produce a wallpaper structure of 442 group symmetry (see figure 1). 


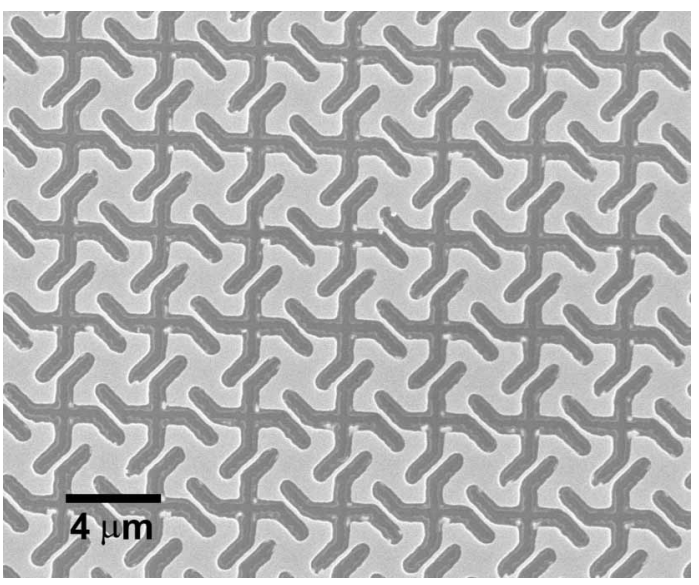

Figure 1. A SEM image of an array of right-handed gammadions etched in a $\mathrm{Ti} / \mathrm{Au} / \mathrm{Ti}$ film on a silicon substrate.

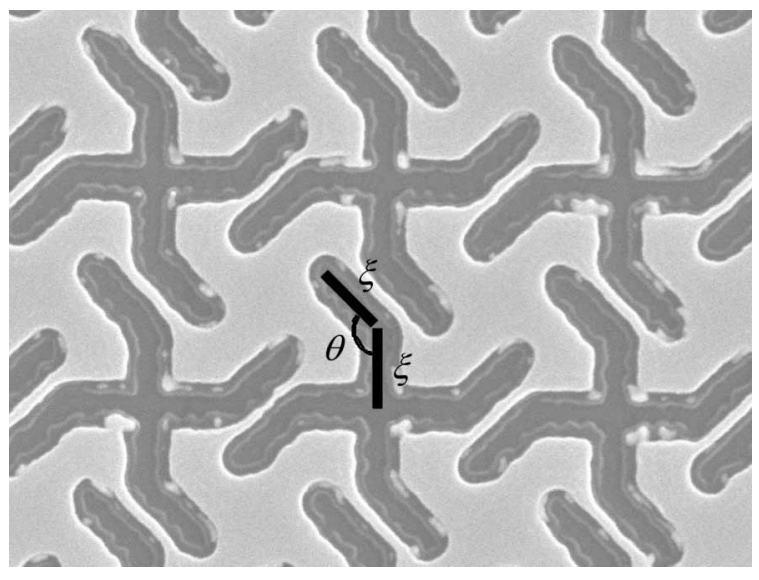

Figure 2. A high magnification SEM image of an array of left-handed gammadions with the internal angle $(\theta)$ and characteristic length $(\xi)$ marked.

Arrays of both clockwise and anticlockwise gammadions, but of otherwise identical design specifications, were fabricated to provide the two enantiomeric forms of the structure, while the 442 symmetry group was chosen because of the absence of any optical birefringence in this structure for light at normal incidence.

The samples themselves were manufactured using a combination of direct-write electron beam lithography and ion beam milling. First a triple layer of metal was deposited by evaporation onto one side of a double-polished silicon wafer. The metal layers were $20 \mathrm{~nm}$ of titanium, followed by $100 \mathrm{~nm}$ of gold, with a final capping layer of $20 \mathrm{~nm}$ of titanium. The first layer of titanium was used to improve the adhesion of the much thicker gold layer to the silicon substrate, while the top layer of titanium was there merely to give the structure a symmetric profile. The metallic trilayer was then patterned by electron beam lithography using a $300 \mathrm{~nm}$ layer of high sensitivity UVIII resist. Arrays of gammadions were patterned into the resist and, after development in MF322 developer, these patterns were etched into the underlying Ti/Au/Ti layer by a process of ion beam milling. This etch process necessarily results in some widening of the legs of the gammadions, together with some etching of the underlying silicon substrate, due its low degree of etching directionality and the absence of a large etch-rate differential between silicon and titanium. The resulting structures therefore consisted of arrays of gammadion-shaped holes in an otherwise planar layer of $\mathrm{Ti} / \mathrm{Au} / \mathrm{Ti}$.

In all of the arrays we have studied, the gammadions had an internal angle, $\theta$, of $135^{\circ}$ and a characteristic length $\xi$ (see figure 2), the pitch of the gratings was $4.0 \mu \mathrm{m}$ in both directions so that the density of gammadions was $6.25 \times 10^{6} \mathrm{~cm}^{-2}$. The area of the arrays available for optical measurements was chosen to be approximately $1.0 \times 1.0 \mathrm{~mm}^{2}$ so that it is much larger than the diameter of the focused laser beam used during optical characterization. As well as investigating the two different enantiomeric forms of the structures, we also looked at the effect of varying their characteristic length, $\xi$, by measuring the properties of three different structures of $\xi=0.8$ $\mu \mathrm{m}, \xi=1.0 \mu \mathrm{m}$, and $\xi=1.4 \mu \mathrm{m}$, while maintaining the same internal angle, $\theta$, and grating period. 


\section{EXPERIMENTAL RESULTS}

The optical characterization of the chiral gratings was performed using a HeNe laser at a wavelength of $632 \mathrm{~nm}$, and the polarization parameters of the diffracted wave were measured using the "rotating wave plate" polarimetric technique. The structures were oriented to the incident beam in such a way that the "plane of incidence" (the plane defined by the incident wave and the normal to the sample) lay parallel to one axis of the gammadion array and perpendicular to the other. As expected, we found that the light scattered from the chiral microstructures showed well-defined rectangular diffraction patterns, but ones where the polarization states of the diffracted waves were noticeably different from those that would be expected for diffraction from a non-chiral grating. In this configuration diffracted waves propagate in both the plane of incidence, and at various angles both above and below it. If the diffracted wave remains in the plane of incidence and the incident wave is polarized either in the plane of incidence (S-polarization) or perpendicular to the plane (P-polarization), no polarization change would be expected for a non-chiral grating as these polarization states represent the eigenstates of the system. However, we found that in the case of our chiral samples the polarization states of the diffracted waves are, in general, elliptical, with the polarization azimuth rotated by some angle to the initial polarization state. In addition, for a given angle of incidence $\beta$, the polarization states of various diffraction orders were found to be different. We have concentrated on characterizing the polarization state of the first-order diffracted wave and measured the difference $\Delta \Theta$ between the polarization azimuth of the incident wave and that of the diffracted wave. We also measured the ellipticity angle $\eta$ of the diffracted wave where $\tan (\eta)$ represents the ratio of the semi-minor and semi-major axes of the ellipse (and hence $\eta=0$ corresponds to a linearly polarized wave, with $\eta= \pm 45^{\circ}$ corresponding right and left circular polarizations accordingly).

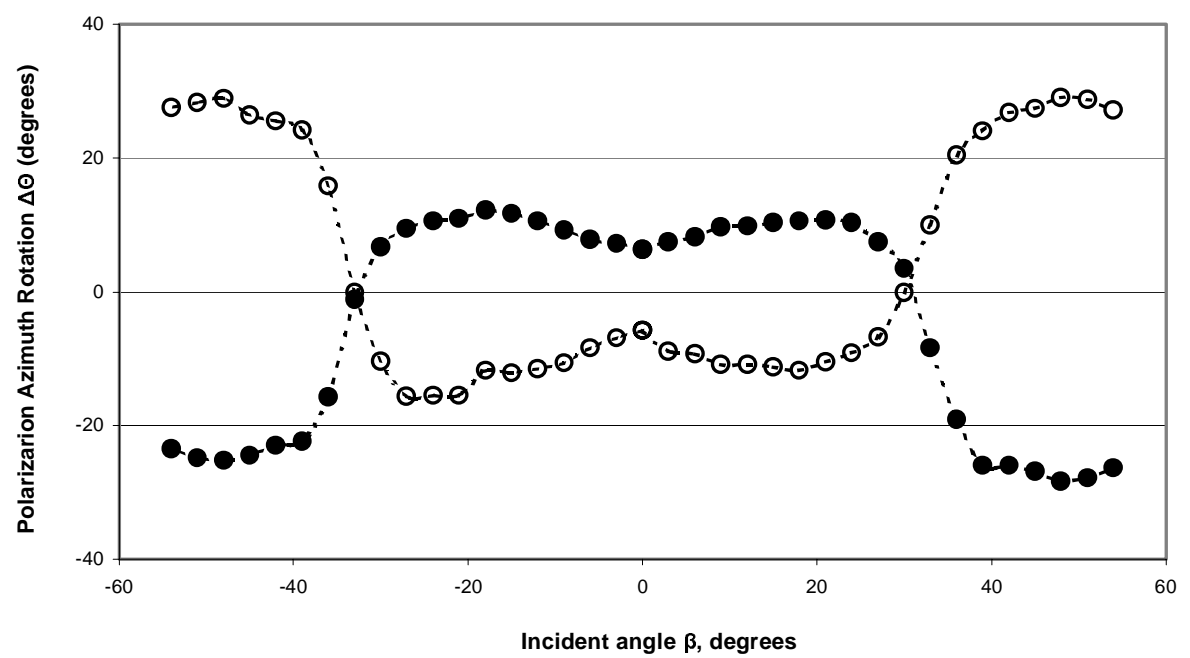

Figure 3. The observed polarization azimuth rotation of light in the first order diffracted beam from an array of gammadions with $\xi=1.4 \mu \mathrm{m}$ as a function of the angle of incidence, $\beta$. The solid circles are the data for clockwise (right-handed) gammadions while the hollow circles are the data for anti-clockwise (left-handed) gammadions. 
We have measured the polarization change of the first order diffracted beam as a function of the angle of incidence, $\beta$, for each of the enantiomeric chiral structures with an internal angle $\alpha=135^{\circ}$ and a characteristic length $\xi=1.4 \mu \mathrm{m}$ for an incident wave that was S-polarized. Figure 3 shows how the polarization azimuth rotation $\Delta \Theta$ varies with angle of incidence, $\beta$, for the two enantiomeric structures, while figure 4 shows the dependence of the degree of ellipticity, $\eta$, again as a function of, $\beta$. Both strongly depend on the angle of incidence, $\beta$. However, it can be seen that whatever the values of $\beta, \Delta \Theta$ and $\eta$, the waves diffracted from each of the two enantiomeric structures were always of the same amplitude, but of strictly opposite sign. We have also compared the polarization azimuth rotation for the same samples for positive and negative incident angles, $\pm \beta$. Although some minor differences in the polarization response for incident angles of opposite signs were observed, the polarization rotation and the ellipticity are found to be largely insensitive to the sign of $\beta$. This is not an obvious result as planar chiral structures configured with positive and negative angles of incidence are not equivalent. Similar results were observed as the value of $\xi$ is reduced to $1.0 \mu \mathrm{m}$ and $0.8 \mu \mathrm{m}$, but the magnitudes of both the polarization azimuth rotation and the ellipticity are reduced.

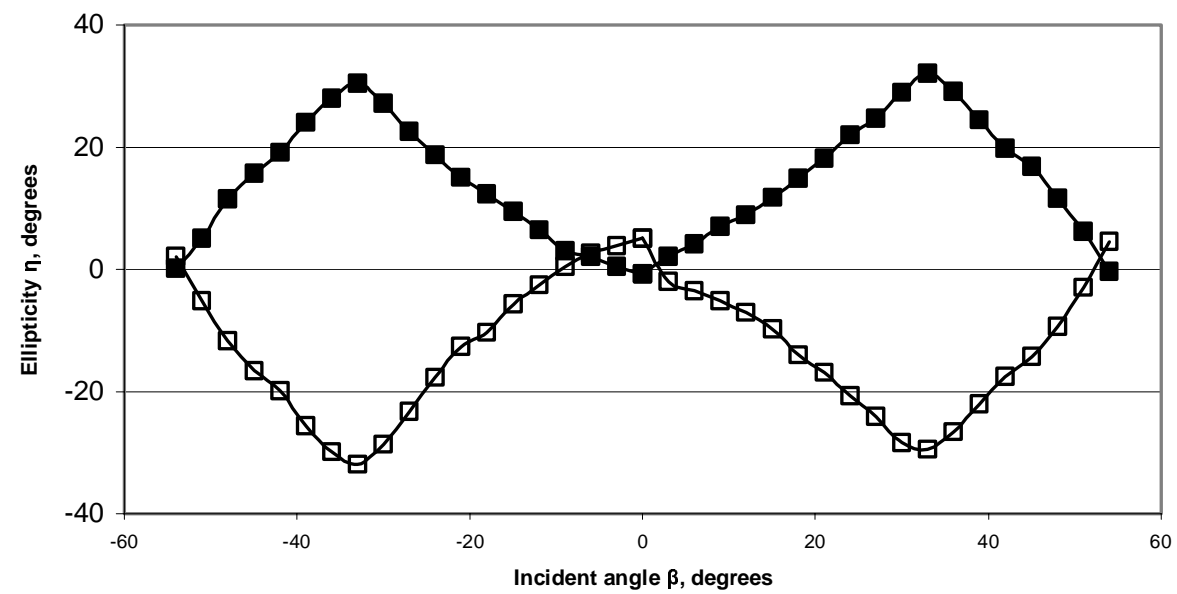

Figure 4. The observed ellipticity of light in the first order diffracted beam from an array of gammadions with $\xi=1.4 \mu \mathrm{m}$ as a function of the angle of incidence, $\beta$. The solid squares are the data for clockwise (right-handed) gammadions while the hollow squares are the data for anti-clockwise (left-handed) gammadions. 


\section{CONCLUSIONS}

Our results appear to show that polarization changes in light diffracted from a chiral planar structure are a true effect of planar chirality. To be a true optical manifestation of two-dimension chirality, the following test must be satisfied: the interaction of light with the two enantiomeric (left-right) forms of the structure must yield different polarization changes, and these changes should not be observed if the structure lacks chirality. More generally, the magnitude of the polarization change and its sign must have a correlation with the geometrical chirality of the structure and, in particular, with its handedness. The results presented in figures 3 and 4 prove that enantiomeric structures do indeed give opposite polarization changes where a non-chiral structure would show no polarization change at all.

\section{ACKNOWLEDGMENTS}

We would like to thank Chong Yew Lee and N.S. Lloyd for experimental assistance.

\section{REFERENCES}

1. P. Le Guennec, J. Math. Phys. 41, 5954 (2000).

2. L.R. Arnaut, J. Electromaget. Wave, 11, 1459 (1997).

3. L.R. Arnaut, L.E. Davis, "Dispersion Characteristics of Planar Chiral Structure", International Conference on Electromagnetics in Advance Applications. September 1215, Torino, Italy, 1995. Proceedings. pp.381-384.

4. Y. Svirko, N. Zheludev and M. Osipov, Appl. Phys. Lett. 78, 498 (2001). 